\title{
Research on Vehicle Dynamic Location Algorithm Based on Interactive Multi Model
}

\author{
Guotong Zhang ${ }^{1}$, Lei Shi ${ }^{2}$ and Jun $\mathrm{Xu}^{3}$ \\ ${ }^{1}$ College of mechanical and electrical automation, Henan polytechnic institute, Henan Nanyang \\ 473000, China \\ ${ }^{2}$ Department of electronic and Information Engineering, Henan polytechnic institute, Henan \\ Nanyang 459000, China \\ ${ }^{3}$ Henan Optical Group Co., Ltd., Henan Nanyang 473000, China
}

Keywords: Vehicle positioning, Dynamic filtering; Kalman filtering, Current statistical model (CSM).

\begin{abstract}
The vehicle positioning accuracy directly related to the dynamic model. For a single dynamic model, it is difficult to describe the state change of the vehicle movement precisely, so this paper proposes a composite model of GPS dynamic positioning system, and this paper designs a vehicle location and tracking algorithm by introducing the adaptive switching mechanism. Comparing the advantages and disadvantages of the Calman filter performance, this paper verifies the effectiveness of the algorithm.
\end{abstract}

\section{Introduction}

According to the number of models adopted by the tracking filter, the maneuvering target tracking method can be divided into the single model tracking and multiple model tracking. The aim of single model tracking is to establish a more ideal maneuvering target model to describe the changing process of target states, namely the change of moving target position, velocity and acceleration $[1,2]$. If the described single motion model matches with tracking the target motion, we can achieve the target tracking. However, in the actual process, the target's motion state is a complex and changeable, we track the maneuvering situation with a single model, and sometimes it will produce a large error.

In land vehicle positioning system, although GPS positioning and navigation system can all-weather, continuous real-time to provide high precision three-dimensional position and velocity information, it is vulnerable to the effects of environmental factors, such as the random signal interference, atmospheric environment error, etc. [3]. In order to improve the accuracy of GPS positioning and reduce the influence of all kinds of random errors, we generally use Kalman filter to remove random errors, which can be best estimated on the true state. However, it is difficult to completely satisfy the assumptions of filter in the application of vehicle positioning. There are two main reasons [4-6]: one is the research research's state and change that are too complex, it is difficult to establish a precise dynamic model; another is the expected value and variance matrix of the Kalman filter's exact dynamic noise statistic characteristic, however in fact they can't be fully known. Therefore, in engineering application, the main problem of Kalman filter application is the establishment of dynamic model and noise.

\section{Description of the Traditional Localization Model}

Target localization technique has been widely used in military and civilian areas, such as the United States has put into use global positioning system (GPS system). The accuracy of system positioning has far-reaching influence for national strategic security. To improve the accuracy, home and abroad has a extensively and in-depth research, so this paper puts forward many different target tracking algorithm [7].

At present, the positioning model has been put into use, there is the two order constant velocity model (CV), three order constant acceleration model (CA), Singer model and current statistical model (CSM), in which current statistical model is a typical representative of the current statistical model. Because of the limitations of these models, filter precision is not high by using a model alone. 
The two order constant velocity model. Assume that the GPS data receiving cycle is $T$, when the vehicle movements are at a constant speed, the state variable will be:

$$
X=\left[x, v_{x}, y, v_{y}\right]^{T} \text {. }
$$

Among them, $x$ and $y$ are the east and north position respectively for the vehicle; $v_{x}$ and $v_{y}$ are the east and north vehicle speed respectively for the vehicle. Then the state equation and e observation equation are:

$$
\begin{aligned}
& X(k+1)=\varphi X(k)+G W(k) . \\
& Z(k)=H X(k)+V(k) .
\end{aligned}
$$

Among them, $\varphi=\left[\begin{array}{llll}1 & T & 0 & 0 \\ 0 & 1 & 0 & 0 \\ 0 & 0 & 1 & T \\ 0 & 0 & 0 & 1\end{array}\right] \quad G=\left[\begin{array}{cc}T / 2 & 2 \\ 1 & 0 \\ 0 & T / 2 \\ 0 & 1\end{array}\right] H=\left[\begin{array}{llll}1 & 0 & 0 & 0 \\ 0 & 0 & 1 & 0\end{array}\right]$ and $W=\left[\begin{array}{ll}w_{1} & w_{2}\end{array}\right]^{T}$. System noise $W$ is the zero mean value, the variance matrix is Gauss random sequence for $Q$, the observation noise $V$ is zero mean and the variance matrix is the white noise of $R$, which is irrelevant with $W$.

Current statistical model. The model is an essentially non-zero time-dependent model, the current probability density of acceleration is shown by the modified Rayleigh, its average values are the predicted values of current acceleration, and the random acceleration is fitting for the related process of first order time in the timeline.

That is:

$$
\begin{aligned}
& \ddot{x}(t)=\bar{a}(t)+a(t) . \\
& a(t)=-\alpha a(t)+\omega(t) .
\end{aligned}
$$

Where, $\bar{a}(t)$ is the current values of the acceleration, it is a constant in the sampling period. The ultimate mathematical expression of CSM model is:

$$
\left[\begin{array}{c}
\dot{x} \\
\ddot{x} \\
x
\end{array}\right]=\left[\begin{array}{ccc}
0 & 1 & 0 \\
0 & 0 & 1 \\
0 & 0 & -a
\end{array}\right]\left[\begin{array}{l}
x \\
\dot{x} \\
\ddot{x}
\end{array}\right]+\left[\begin{array}{l}
0 \\
0 \\
a
\end{array}\right] \bar{a}(t)+\left[\begin{array}{l}
0 \\
0 \\
1
\end{array}\right] \omega(t) \text {. }
$$

The model can truly reflect the scope and intensity change of target maneuver, which can be better to respond target maneuvering situation without testing.

\section{The Vehicle Location Algorithm Based on Compound Model}

During the vehicles in motion, there are two types of movement states generally: long time uniform motion and frequent variable speed maneuver, because the single model is difficult to accurately describe vehicle motion characteristics.

The CV model can track the moving target well. The CSM model can track maneuvering target well, but it is not good for the non-maneuvering target tracking. In view of the vehicle motion features, combined with CV and the CSM model, this paper proposes a vehicle locating and tracking algorithm based on compound model, and it can carry out adaptive model switching according to the running state of the vehicle, to improve the tracking accuracy effectively.

Algorithm description. The vehicle shifts from the uniform speed to vibrational acceleration maneuvering situation. In uniform cases, when the tracking regional stability, there are: 


$$
\dot{x} \frac{k}{k} \rightarrow\left|x \frac{k}{k}-x \frac{k-1}{k-1}\right| .
$$

The current speed and vehicle distance per unit time are linear relationship. But

$$
|\Delta \dot{x}(k / k)|<T h_{1} \text {. }
$$

Among them, $T h_{1}$ is the tracking error threshold, it can be considered that the vehicle is still in the process of uniform motion, so we can observe the change of the vehicle distance, to determine whether the vehicle is in the motor. In the error case, it is considered that the speed will be a certain fluctuation in a neighborhood of the current speed. Experimental results show that we can be used as a vehicle to determine the mobility threshold when $T h_{1}=5 \% \dot{x}(k / k)$. Of course, there is to enter uniform motion state soon after the vehicle gear, Due to observe some time $T_{1}$ when $|\Delta \dot{x}(k / k)|>T h_{1}$ continuously, we can determine the vehicle that has entered the motor state, so we can determine the vehicle's motion state according to experience to take $T_{1}=10 \mathrm{~s}$.

Due to error is larger when the CSM model track the uniform target, if you use it to access predictive value, according to (5) and (6) to determine the status of the vehicle, there is a big error. This paper judges the vehicles in motion, it will use CV and CSM model to estimate respectively, however, subject to CSM model estimation, CV estimation only as a reference, to determine whether the vehicle into the uniform state. Here to observe some time $T_{2}$ when the results of CV estimation to satisfy (6), we can judge the vehicle entered the uniform state. Due to CV estimates error, in uniform cases, take some time to adjust, take $\mathrm{T}_{2}=20 \mathrm{~S}$.

Calculation. (1) Using CV and CSM model to track vehicle,with CSM estimate results base. Respectively recorded the value of $|\Delta \dot{x}(k / k)|$ which in each sample point under CV model, when observing results meet (6), some time continuously $\mathrm{T}_{2}$, into (step 2); otherwise, repeat steps (1) operations.

(2) Using CV model to track vehicle, respectively recorded the value of $|\Delta \dot{x}(k / k)|$ which in each sample point, observe some time continuously $T_{1}$ when $|\Delta \dot{x}(k / k)|>T h_{1}$, into (step 1); otherwise, repeat steps (2) operations.

\section{Experimental Analysis}

In order to check the algorithm is practical, collected a vehicle driving track,respectively use CSM model and the algorithm to processing and filtering. In order to simplify the calculation, vehicles along the $x$ axis movement, and experimental results as follows.

In Figure 1, target initial state is 0 points, starting speed is $5 \mathrm{~m} / \mathrm{s}$, the initial covariance is 10 , acceleration $0.2 \mathrm{~m} / \mathrm{s}^{2}$, moving forward 50s, uniform driving 50s, then moving forward 50s with the acceleration as $-0.2 \mathrm{~m} / \mathrm{s}^{2}$. The sampling period $\mathrm{T}=1 \mathrm{~s}$, the experimental results are shown in Figure 1 , the further calculation shows that CSM model error is 5.43, and the algorithm is 5.13. 


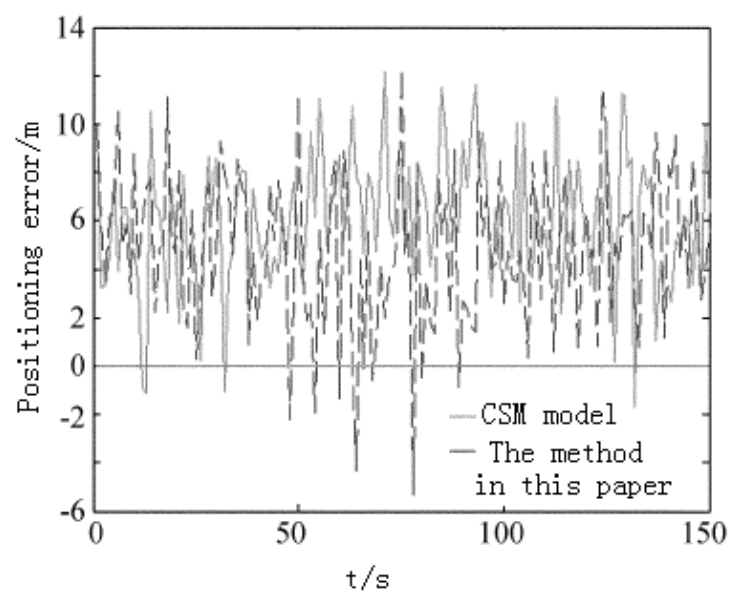

Fig. 1 Test 1 positioning distance error chart

In Figure 2, target initial state is 0 points, starting speed is $5 \mathrm{~m} / \mathrm{s}$, the initial covariance is 10 , acceleration $0.2 \mathrm{~m} / \mathrm{s}^{2}$, moving forward 30s, moving forward $20 \mathrm{~s}$ with the acceleration as $-0.1 \mathrm{~m} / \mathrm{s}^{2}$, then uniform driving 50s; then moving forward 50s with the acceleration as $-0.2 \mathrm{~m} / \mathrm{s}^{2}$; then moving forward 50s with the acceleration as $-0.3 \mathrm{~m} / \mathrm{s}^{2}$. The sampling period $\mathrm{T}=1 \mathrm{~s}$, experimental results are shown in Figure 2, the further calculation shows that CSM model error is 5.41, and the algorithm is 5.24 .

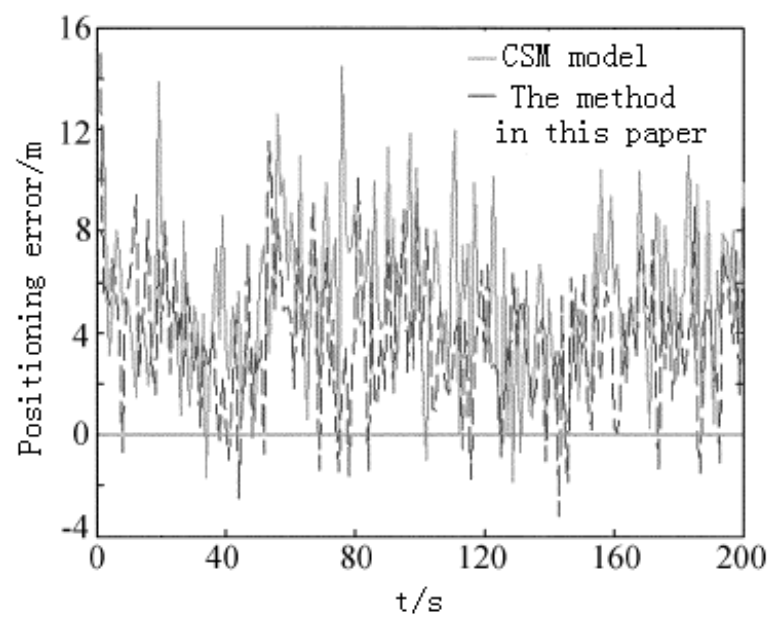

Fig.2 Test 2 positioning distance error chart

\section{Summary}

Establishing effective vehicle movement model and adopting appropriate algorithm, it is very important for vehicles location and tracking. After studying the CV model, CSM model based on the filtering characteristics, for the vehicles occurred during the different states, achieved the adaptive switching between the CV model and CSM model, and using the Kalman filter to predict and projection the vehical location, in order to achieve accurate and effective location tracking. Experimental results show that the algorithm can effectively improve the tracking accuracy.

\section{References}

[1] H.N. Wang. GPS navigation and application. Beijing: science press, 2013:106-121.

[2] Y.Q. Shen, H.J. Gong, Y. Xiong. Application of adaptive extended Kalman filiter for tracking a moving target. Computer Simulation, 2014(2): 210-213.

[3] Q. Fang, X.C. Mei, Y.P. Zhang. Design of Kalman filter for maneuvering target track. Radar science and technology, 2015, 4(1): 49-55. 
[4] Z. Chen, X. Yuan. A federated Kalman filter and its application at GPS integrated navigation system. Chongqing University, 2015, 28(10): 1-5.

[5] Y.H. Li. Improve on fast tracking maneuver targets for the current statistical model. Intelligence command and control systems and simulation technology, 2014, 26(6): 38-43.

[6]J.H. Yu, L. Wang. A vehicle tracking and locating algorithm based on improved Camshift combined with Kalman filter. Computer Knowledge and Technology, 2016, 12(8): 163-163.

[7] D.L. Shi, P.J. Ye, Y. Jia. Character points matching method on vehicle stereo vision navigation. Chinese Journal of agricultural machinery, 2014, 38(9): 134-137. 